\title{
Rearrangeable and Repackable S-W-S Elastic Optical Networks for Connections with Limited Bandwidths
}

\author{
Bey-Chi Lin $\mathbb{D}$
}

Department of Applied Math, National University of Tainan, Tainan City 70005, Taiwan;

beychi@mail.nutn.edu.tw; Tel.: +886-6-2133-111

Received: 30 December 2019; Accepted: 9 February 2020; Published: 13 February 2020

\begin{abstract}
Elastic optical networks flexibly allocate bandwidth to a connection for improving utilization efficiency. The paper considers an optical node architecture that is similar to a three-stage Clos network for elastic optical networks. The architecture, which employs space switching in the first and the third stages and wavelength switching in the second stage, is called an S-W-S switching fabric. In this paper, we propose a graph-theoretic approach and different routing algorithms to derive the sufficient conditions under which an S-W-S switching fabric will be rearrangeable nonblocking and repackable nonblocking. The proposed rearrangeable and repackable nonblocking S-W-S switching fabrics for connections with limited bandwidths consume around half the number of middle wavelength switches compared to strictly nonblocking S-W-S switching fabrics.
\end{abstract}

Keywords: elastic optical network; rearrangeable nonblocking; space-wavelength-space switching fabric; repackable nonblocking

\section{Introduction}

Elastic optical networks (EONs) [1-3] are a technique introduced to support future high-speed optical networking and rapid growth of Internet traffic. EONs provide superior flexibility and scalability in spectrum allocation. To improve spectrum allocation efficiency, EONs use the flexible frequency grid proposed by the International Telecommunication Union [4]. In EONs, the spectrum is currently divided into several $12.5 \mathrm{GHz}$ fixed-width frequency slots called frequency slot units (FSUs) [4]. An optical connection that requires bandwidths greater than $12.5 \mathrm{GHz}$ can spread over a number of, say $m$, adjacent FSUs, and it is called an $m$-slot connection. Usually, $m$ is upper bounded by a value, denoted by $m_{\max }$.

Various switching fabrics for EONs have been proposed [5-8]. One of them is the three-stage wavelength-space-wavelength (W-S-W) architecture, which adopts spectrum converters in the first and last stages and space switching in the middle stage [8]. A demonstration of a $16 \times 16 \mathrm{~W}-\mathrm{S}-\mathrm{W}$ optical switching test bed for EONs has been presented in [6]. This architecture with different types of nonblockingness was considered in [8-12]. Another three-stage switching fabric for EONs is the space-wavelength-space (S-W-S) architecture, which has only spectrum converters in the middle stage [7]. Both W-S-W and S-W-S architectures can be used as the optical circuit switching part, which serves elephant traffic between servers, of a datacenter network $[7,13]$. The S-W-S architecture generally consumes fewer middle switches than W-S-W architectures [8,9], especially when $m_{\max }$ is growing. Moreover, unlike the W-S-W architecture, the S-W-S architecture can be practically realized for switching fabrics when a larger $m_{\max }$ and a greater number of FSUs are used in one input or output. In this paper, we consider one S-W-S architecture, called an SWS1 switching fabric.

In EONs, an $m$-slot connection is set up between $m$ free FSUs in the input and output fibers. Thus, the switching fabrics can never be blocked because of a lack of free FSUs at the input and output fibers. Switching fabrics can be of various types of nonblockingness, three of which are strictly nonblocking 
(SNB) [14,15], rearrangeable nonblocking (RNB) [14,15], and repackable nonblocking (RPNB) [16-19]. In an SNB switching fabric, a connection will never be blocked by existing connections. In an RNB switching fabric, the nonblocking state can be kept by rearranging existing connections upon the arrival of a connection, while in an RPNB switching fabric, rearranging existing connections is done upon the departure of a connection. It is worth noting that time is less critical for connection rearrangement during departures than during arrivals. An alternate definition of an RNB switching fabric is that a set (or frame) of connections can be routed simultaneously.

Sufficient and necessary conditions for an SNB SWS1 switching fabric were derived and proved in [7]. In this paper, we use two different approaches to generate RNB SWS1 switching fabrics for $m$-slot connections for $1 \leq m \leq m_{\max }$ with $m_{\max }=1$ and $2 \leq m_{\max } \leq n$, respectively, where $n$ is the number of FSUs in each link of the SWS1 switching fabric. A similar approach to that for an RNB SWS1 switching fabric with $2 \leq m_{\max } \leq n$ is also applied to generate an RPNB SWS1 switching fabric with $2 \leq m_{\max } \leq n$. The proposed RNB and RPNB SWS1 switching fabrics for connections with a limited $m_{\max }$ consume around half the number of middle wavelength switches than the SNB SWS1 switching fabric.

The remainder of the paper is organized as follows. In Section 2, we introduce the SWS1 switching fabric and earlier results. The sufficient and necessary conditions under which an SWS1 switching fabric with $m_{\max }=1$ will be RNB are derived in Section 3. The sufficient conditions under which an SWS1 switching fabric with $2 \leq m_{\max } \leq n$ will be, respectively, RNB and RPNB are derived in Section 4 . Conclusions are provided in Section 5.

\section{Preliminaries}

The SWS1 switching fabric considered in this paper, represented as $\operatorname{SWS1}(q, p, n)$, is given in Figure 1. It uses bandwidth-variable space switches (BV-SSs) in stages 1 and 3, and bandwidth-variable wavelength-converting switches (BV-WSs) in stage 2. The BV-SS has no spectrum conversion capability, and the BV-WS has full-range conversion capability. Specifically, in a BV-SS, an $m$-slot connection, which uses $m$ adjacent FSUs in the input, must be switched to the $m$ adjacent FSUs of the same nominal frequencies in the output; in a BV-WS, an $m$-slot connection, which uses $m$ adjacent FSUs in the input, can be switched to any other $m$ adjacent FSUs in the output. The internal architectures of a BV-SS and a BV-WS can be found in [7].

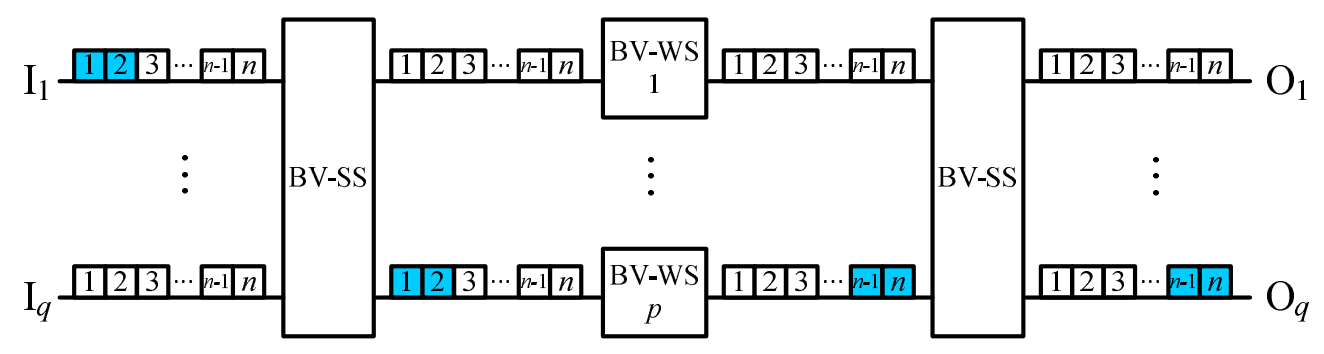

Figure 1. An $\operatorname{SWS1}(q, p, n)$ switching fabric.

In the SWS1 $(q, p, n)$ switching fabric (Figure 1), the first (or input) stage contains one BV-SS of capacity $q \times p$. The middle stage contains $p$ BV-WSs, each of capacity $1 \times 1$. The last (or output) stage contains one BV-SS of capacity $p \times q$. Each link has $n$ FSUs, which are numbered from $1 . \mathrm{I}_{i}\left(\right.$ or $\left.\mathrm{O}_{i}\right)$ represents the $i$ th input (or output) for $1 \leq i \leq q$, and $\mathrm{I}^{\prime}{ }_{j}$ ( ${\text { or } \mathrm{O}_{j}^{\prime}}_{j}$ ) represents the input (or output) of the $j$ th BV-WS for $1 \leq j \leq p$. We also call $\mathrm{I}^{\prime} j$ and $\mathrm{O}_{j}^{\prime}$ internal links.

The SWS1 switching fabric serves $m$-slot connections. An $m$-slot connection is represented as $(i, j, m)$ if it is from $\mathrm{I}_{i}$ and destined to $\mathrm{O}_{j}$. In order to route each $(i, j, m)$ in an $\operatorname{SWS} 1(q, p, n)$ switching fabric, we have to find two internal links $\mathrm{I}_{k}{ }_{k}$ and $\mathrm{O}^{\prime}{ }_{k}$ where $1 \leq k \leq p$, which have $m$ adjacent FSUs, using the same nominal frequencies, available for $(i, j, m)$ in the input $\mathrm{I}_{i}$ and output $\mathrm{O}_{j}$, respectively (see Figure 1). That is, we have to find two links, $\mathrm{I}^{\prime}{ }_{k}$ and $\mathrm{O}^{\prime}{ }_{k}$, such that there are $m$ available common 
adjacent FSUs of links $\mathrm{I}_{i}$ and $\mathrm{I}^{\prime}{ }_{k}\left(\right.$ or $\mathrm{O}_{j}$ and $\mathrm{O}^{\prime}{ }_{k}$ ). When two such internal links can be found, the SWS1 $(q$, $p, n)$ switching fabric is nonblocking to $(i, j, m)$; otherwise, $(i, j, m)$ is blocked.

Sufficient and necessary conditions under which an $\operatorname{SWS1}(q, p, n)$ switching fabric will be SNB were derived in [7], which showed that an $\operatorname{SWS1}(q, p, n)$ switching fabric for $m$-slot connections with $1 \leq m \leq m_{\max }$, is SNB if and only if

$$
p \geq \begin{cases}2 m_{\max } \cdot(q-1)+1, & \text { for } m_{\max } \leq \frac{n q}{2 q-1}, \\ n q-\frac{n q}{2 q-1}+1, & \text { for } m_{\max } \geq \frac{n q}{2 q-1}+1 .\end{cases}
$$

In this paper, two different approaches are proposed to generate RNB SWS1 $(q, p, n)$ switching fabrics for $m$-slot connections where $1 \leq m \leq m_{\max }$ with $m_{\max }=1$ and $2 \leq m_{\max } \leq n$, respectively. A similar approach to that for generating $\operatorname{RNB~SWS1}(q, p, n)$ switching fabrics with $2 \leq m_{\max } \leq n$ is also applied to generate an $\operatorname{RPNB} \operatorname{SWS} 1(q, p, n)$ switching fabric with $2 \leq m_{\max } \leq n$.

\section{Rearrangeable Nonblocking (RNB) SWS1 $(q, p, n)$ Switching Fabrics with $m_{\max }=1$}

Suppose a frame $F$ of connections is given in an $\operatorname{SWS} 1(q, p, n)$ switching fabric for $m$-slot connections with $1 \leq m \leq m_{\max }$ (Figure 2a). The routing of connections includes two parts: (1) Internal link selection and (2) FSU assignment. In this section, we propose a graph-theoretic approach to study the FSU assignment in the case of $m_{\max }=1$. We construct a graph $G_{F}$ in which each left vertex $i$ (or right vertex $j$ ) denotes an $\mathrm{I}_{i}$ (or $\mathrm{O}_{j}$ ) and an edge between two vertexes $i$ and $j$ denotes a connection $(i, j, 1)$ in frame $F$ (Figure $2 b$ ). Obviously, $G_{F}$ is a bipartite graph. Here, we use $\Delta\left(G_{F}\right)$ to denote the maximum degree of $G_{F}$. Since each link is assigned $n$ FSUs, it can carry at most $n 1$-slot connections. This leads to $\Delta\left(G_{F}\right) \leq n$. According to graph theory [20], since $G_{F}$ is a bipartite graph we have that $G_{F}$ is n-edge colorable and use colors $1,2, \ldots, n$ for its edge coloring. We propose Routing Algorithm 1, given in the following, to route each $(i, j, 1)$ in frame $F$ for RNB conditions [14].

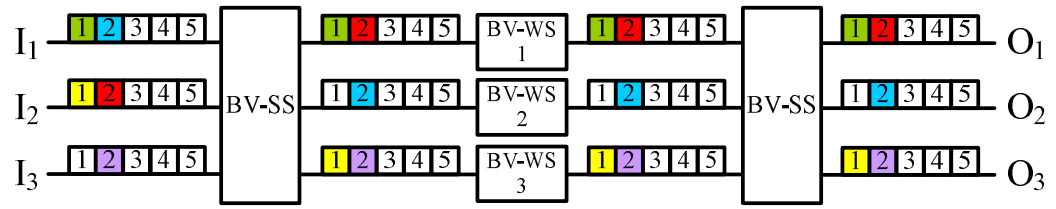

(a)

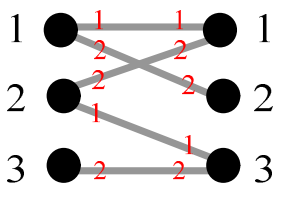

(b)

Figure 2. A frame $F$ consisting of connections $(1,1,1),(1,2,1),(2,3,1),(2,1,1)$, and $(3,3,1)$ is given in an SWS1 $(3,3,5)$ switching fabric. (a) A routing of connections in F under Routing Algorithm 1 referring to the edge-coloring given in (b). (b) The corresponding graph $G_{F}$ of (a), in which colors 1 and 2 (marked in red font) are adopted for edge-coloring.

\section{Routing Algorithm 1:}

Each connection $(i, j, 1)$ is routed in the $c$ th FSU of links $\mathrm{I}_{i}, \mathrm{I}^{\prime}{ }_{j}, \mathrm{O}^{\prime}{ }_{, j}$ and $\mathrm{O}_{j}$, if the corresponding edge of $(i, j, 1)$ in graph $G_{F}$ is assigned color $c$.

As we can see in Routing Algorithm 1, output $j$ determines the selection of internal links, i.e., $\mathrm{I}^{\prime}{ }_{j}$ and $\mathrm{O}^{\prime}{ }_{j}$, and the FSU assignment is determined by the edge coloring of $G_{F}$. An example is given in Figure 2, where a frame $F$ consisting of connections $(1,1,1),(1,2,1),(2,3,1),(2,1,1)$, and $(3,3,1)$ is routed in an SWS1 $(3,3,5)$ switching fabric according to Routing Algorithm 1 . Next, in Theorem 1, we derive the sufficient and necessary conditions under which an $\operatorname{SWS} 1(q, p, n)$ switching fabric is RNB for 1-slot connections.

Theorem 1. An SWS1 $(q, p, n)$ switching fabric for 1-slot connections is RNB if and only if $p \geq q$. 
Proof. The necessary condition is true when each input $\mathrm{I}_{i}$ holds $q$ connections $(i, i, 1)$, each of which is routed in internal links $\mathrm{I}_{i}^{\prime}$ and $\mathrm{O}^{\prime}{ }_{i}$ according to Routing Algorithm 1 , for $1 \leq i \leq q$. Next, we consider the sufficient condition. From the design of Routing Algorithm 1, we can see that all connections destined to the same output, say $\mathrm{O}_{j}$, are routed in the same internal links $\mathrm{I}_{j}^{\prime}$ and $\mathrm{O}^{\prime}{ }_{j}$. Since each output has $n$ FSUs, at most $n 1$-slot connections can be destined to the same output link. Thus, at most $n 1$-slot connections can be assigned to each internal link, which occupies $n$ FSUs. Recall that for each frame $F$ of connections given in an $\operatorname{SWS} 1(q, p, n)$ switching fabric, the corresponding graph $G_{F}$ is $n$-edge colorable. This implies that there exists an FSU assignment, i.e., a nonblocking routing, to rearrange all connections assigned in the same link by Routing Algorithm 1. Thus, an $\operatorname{SWS1}(q, p, n)$ switching fabric is RNB if its number of internal links, i.e., $p$, is sufficient to carry all connections. Recall that each connection $(i, j, 1)$ is held by internal links $\mathrm{I}_{j}^{\prime}$ and $\mathrm{O}_{j}^{\prime}$ (Routing Algorithm 1 ), and an $\operatorname{SWS1}(q, p, n)$ switching fabric has only $q$ output links. Therefore, $q$ internal links per stage, i.e., $p \leq q$, are sufficient for the $\operatorname{SWS1}(q, p, n)$ switching fabric to be RNB.

For an $\operatorname{SWS1}(q, p, n)$ switching fabric with given parameters $q$ and $n$, reducing the value of $p$, namely, the number of BV-WSs in stage 2, leads to lowering the hardware cost, area requirements, and power consumption. Theorem 1 shows that the upper bound of the proposed $\operatorname{RNB} \operatorname{SWS} 1(q, p, n)$ switching fabric for 1-slot connections reduces by around half the number of middle switches compared to the number in the SNB SWS1 $(q, p, n)$ switching fabric given in [7]. It is worth noting that the left $q \times p$ BV-SS of the SWS1 $(q, p, n)$ switching fabric for 1-slot connections is RNB as well if and only if $p \geq q$. This is true since each connection $(i, j, 1)$ is routed in the $c$ th FSU of links $\mathrm{I}_{i}$ and $\mathrm{I}^{\prime}{ }_{j}$ if color $c$ is assigned for its corresponding edge in graph $G_{F}$. In other words, an $\operatorname{RNB} \operatorname{SWS1}(q, p, n)$ switching fabric for 1-slot connections can be reduced to a $q \times q$ BV-SS.

\section{RNB and RPNB SWS1 $(q, p, n)$ Switching Fabrics with $2 \leq m_{\max } \leq n$}

\subsection{RNB SWS1 $(q, p, n)$ switching fabrics with $2 \leq m_{\max } \leq n$}

Recall that an RNB switching fabric is defined as one in which each frame of connections given in the switching fabric can be routed simultaneously. We considered this definition in Section 3. An alternate definition of an RNB switching fabric is one in which the nonblocking state can be maintained by rearranging existing connections upon the arrival of a connection. Note that this definition, as with the RPNB switching fabric, considers connections that arrive and depart the switching fabric sequentially. In order to extend the RNB results for $2 \leq m_{\max } \leq n$, which will be given in Theorem 2 later, to easily being RPNB (see Section 4.2 for details), in this section, we consider the RNB switching fabric given by the alternate definition. That is, we route each connection sequentially as it arrives in the switching fabric. In addition, the connection which requires rearrangement has to be rearranged not only inside the switching fabric but also in the input and the output links. Given an $\operatorname{SWS1}(q, p, n)$ switching fabric for $m$-slot connections where $1 \leq m \leq m_{\max }$ with $2 \leq m_{\max } \leq n$, we partition all internal links in each stage into $q$ groups numbered from 1 , each of which consists of $q$ internal links (see Figure 3a), and allow the $i$ th link of group $j$, i.e., $\mathrm{I}_{q \cdot(j-1)+i}$ and $\mathrm{O}_{q \cdot(j-1)+i}^{\prime}$, to carry only connections from input $\mathrm{I}_{i}$ and destined to output $\mathrm{O}_{j}$. That is, connections from the same input can be carried by $q$ internal links per stage according to their destinations, and connections carried in the same internal link are destined to the same output. Note $q^{2}$ internal links, i.e., $p=q^{2}$, are required in each link stage under this scheme. We use the algorithm shown in the following to set up each arrival connection $(i, j, m)$. 


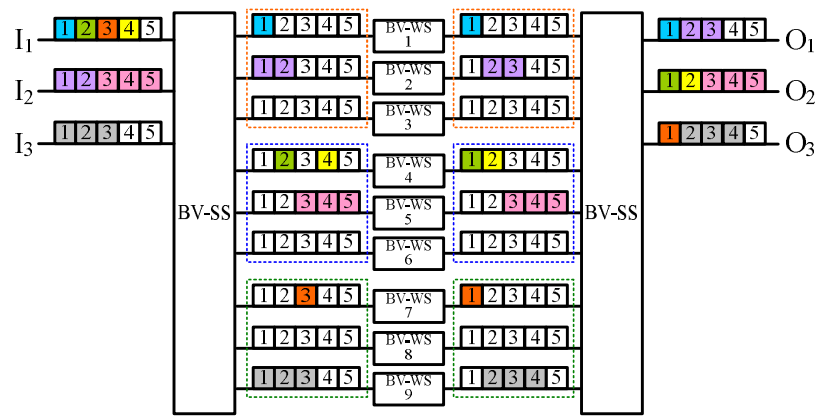

(a)

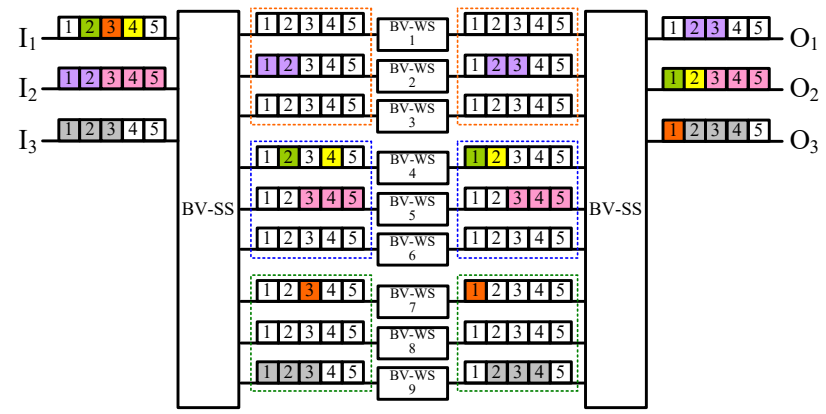

(b)

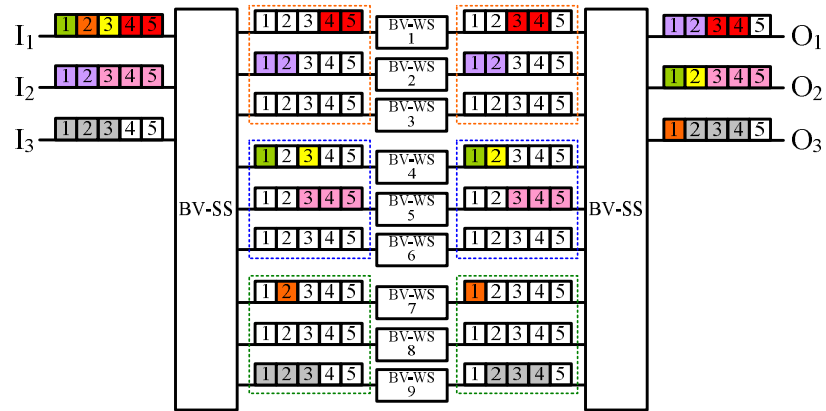

(c)

Figure 3. Suppose connections $(1,1,1),(1,2,1),(1,3,1),(1,2,1),(2,1,2),(2,2,3)$, and $(3,3,3)$ arrive sequentially in an $\operatorname{SWS1}(3,9,5)$ switching fabric. Note each dashed box is a group of internal links. (a) These connections are routed by Routing Algorithm 2 (for Arrivals). (b) Suppose connection $(1,1,1)$ departs, and $(1,1,2)$ arrives. Then, connection $(1,1,2)$ is blocked. (c) Connections marked in green, orange, yellow, and purple are rearranged according to Routing Algorithm 2 (for Rearrangements), and $(1,1,2)$ can be routed in the SWS1 $(3,9,5)$ switching fabric.

\section{Routing Algorithm 2 (for Arrivals):}

Step 1: $(i, j, m)$ is routed in the leftmost $m$ adjacent available FSUs of $\mathrm{I}_{i}$ and the same FSUs of $\mathrm{I}_{q \cdot(j-1)+i}^{\prime}$. Step 2: $(i, j, m)$ is routed in the leftmost $m$ adjacent available FSUs of $\mathrm{O}_{j}$ and the same FSUs of $\mathrm{O}_{q \cdot(j-1)+i}^{\prime}$.

Figure 3a gives an example, where connections $(1,1,1),(1,2,1),(1,3,1),(1,2,1),(2,1,2),(2,2,3)$, and $(3,3,3)$ arrive in an SWS1 $(3,3,5)$ switching fabric sequentially and Routing Algorithm 2 (for Arrivals) is applied.

Suppose Routing Algorithm 2 (for Arrivals) is applied on an SWS1 $(q, p, n)$ switching fabric for $m$-slot connections where $1 \leq m \leq m_{\max }$ with $2 \leq m_{\max } \leq n$, and no existing connection departs. In Property 1, we derive the sufficient condition under which an $\operatorname{SWS1}(q, p, n)$ switching fabric will be nonblocking. 
Property 1. Suppose Routing Algorithm 2 (for Arrivals) is applied on an SWS1 $(q, p, n)$ switching fabric for $m$-slot connections where $1 \leq m \leq m_{\max }$ with $2 \leq m_{\max } \leq n$. If connections are only allowed to be added into the switching fabric and no existing connections can be deleted, then the switching fabric will never go into a blocking state if $p \geq q^{2}$.

Proof. As we can see from Routing Algorithm 2 (for Arrivals), connections assigned in each link of an SWS1 $(q, p, n)$ switching fabric occupy (at most $n$ ) consecutive FSUs from FSU 1 if no connection departs. Since each link has $n$ FSUs, the $m$ available common adjacent FSUs of $\mathrm{I}_{i}$ and $\mathrm{I}_{q \cdot(j-1)+i}$ (or $\mathrm{O}_{j}$ and $\left.\mathrm{O}_{q \cdot(j-1)+i}^{\prime}\right)$ can always be found for each arrival connection $(i, j, m)$ if no connection departs. In addition, connections from the same input are routed in $q$ internal links per stage according to their destinations (Routing Algorithm 2 (for Arrivals)), and there are $q$ input links. Thus, at most $q^{2}$ internal links, i.e., $p \geq q^{2}$, are required for an $\operatorname{SWS1}(q, p, n)$ switching fabric to be nonblocking if no connection departs.

Once an existing connection departs, the $m$ available common adjacent FSUs of $\mathrm{I}_{i}$ and $\mathrm{I}_{q \cdot(j-1)+i}$ (or $\mathrm{O}_{j}$ and $\left.\mathrm{O}_{q \cdot(j-1)+i}^{\prime}\right)$ may not be found for some connection $(i, j, m)$. This may lead to a blocking state, and rearrangements are required. Figure $3 \mathrm{~b}$ gives an example, where after connection $(1,1,1)$ departs, $(1,1,2)$ arrives and is blocked since no two available common adjacent FSUs of links $\mathrm{I}_{1}$ and $\mathrm{I}^{\prime}{ }_{1}$ can be found. Suppose $(i, j, m)$ arrives in the switching fabric and it is blocked. In order to maintain a nonblocking state, all existing connections held in links $\mathrm{I}_{i}$ and $\mathrm{O}_{j}$ are packed together, and they are rearranged from the left side of $\mathrm{I}_{i}$ and $\mathrm{O}_{j}$; that is, all of these connections use the leftmost adjacent FSUs of $\mathrm{I}_{i}$ and $\mathrm{O}_{j}$, such that the remaining free FSUs of links $\mathrm{I}_{i}$ and $\mathrm{O}_{j}$ are still consecutive and available for $(i, j, m)$ (Figure $3 c)$. Once a blocking state occurs due to $(i, j, m)$, the algorithm given below is adopted to rearrange all of the existing connections held in $\mathrm{I}_{i}$, say $\left(i, j^{\prime}, m\right)$, and $\mathrm{O}_{j}$, say $\left(i^{\prime}, j, m\right)$ for $1 \leq i, i^{\prime}, j, j^{\prime} \leq q$.

\section{Routing Algorithm 2 (for Rearrangements):}

Step 1: All connections held in $\mathrm{I}_{i}$, that is, $\left(i, j^{\prime}, m\right)$ for $1 \leq j^{\prime} \leq q$, are rearranged to the leftmost $m$ adjacent available FSUs of $\mathrm{I}_{i}$ and the same FSUs of $\mathrm{I}_{q \cdot\left(j^{\prime}-1\right)+i}$.

Step 2: All connections held in $\mathrm{O}_{j}$, that is, $\left(i^{\prime}, j, m\right)$ for $1 \leq i^{\prime} \leq q$, are rearranged to the leftmost $m$ adjacent available FSUs of $\mathrm{O}_{j}$ and the same FSUs of $\mathrm{O}_{q \cdot(j-1)+i^{\prime}}^{\prime}$.

Figure $3 c$ gives an example where the arrival connection $(1,1,2)$ can be routed in the SWS1 $(3,9,5)$ switching fabric after all existing connections carried in links $\mathrm{I}_{1}$ and $\mathrm{O}_{1}$ are rearranged by Routing Algorithm 2 (for Rearrangements).

Suppose Routing Algorithm 2 (for both Arrivals and Rearrangements) is used on an SWS1 $(q, p, n)$ switching fabric for $m$-slot connections where $1 \leq m \leq m_{\max }$ with $2 \leq m_{\max } \leq n$. In Theorem 2, we derive the sufficient condition under which the $\operatorname{SWS} 1(q, p, n)$ switching fabric will be RNB.

Theorem 2. An $\operatorname{SWS1}(q, p, n)$ switching fabric for $m$-slot connections, $1 \leq m \leq m_{\max }$ and $2 \leq m_{\max } \leq n$, is RNB if $p \geq q^{2}$.

Proof. Routing Algorithm 2 (for Arrivals) guarantees an $\operatorname{SWS1}(q, p, n)$ switching fabric for $m$-slot connections where $1 \leq m \leq m_{\max }$ with $2 \leq m_{\max } \leq n$ is nonblocking and all connections carried on each link occupy adjacent FSUs from FSU 1 if $p \geq q^{2}$ and no connection departs (Property 1). Recall that once an existing connection departs, connections carried on some links may not remain consecutive, and this implies that the $m$ available common adjacent FSUs of $\mathrm{I}_{i}$ and $\mathrm{I}_{q \cdot(j-1)+i}\left(\right.$ or $\mathrm{O}_{j}$ and $\left.\mathrm{O}_{q \cdot(j-1)+i}^{\prime}\right)$ may not be found for a connection $(i, j, m)$. However, Routing Algorithm 2 (for Rearrangements) rearranges all inconsecutive connections such that all of them are repacked in adjacent FSUs from FSU 1. This leads to $p \geq q^{2}$ being sufficient for an $\operatorname{RNB} \operatorname{SWS1}(q, p, n)$ switching fabric with $2 \leq m_{\max } \leq n$ under Routing Algorithm 2 (for Arrivals and Rearrangements) even if existing connections depart. Thus, this theorem, which follows from applying Routing Algorithm 2, is true. 
Theorem 2 shows the proposed $\operatorname{RNB} \operatorname{SWS1}(q, p, n)$ switching fabric with $2 \leq m_{\max } \leq n$ requires fewer middle switches than the SNB SWS1 $(q, p, n)$ switching fabric given in [7] as $\frac{q^{2}-1}{2(q-1)} \leq m_{\max } \leq \frac{n q}{2 q-1}$ (Equation (1)). Thus, the upper bound of the proposed RNB SWS1 $(q, p, n)$ switching fabric for $m$-slot connections where $1 \leq m \leq m_{\max }$ with $2 \leq m_{\max } \leq n$ reduces by around half the number of middle switches relative to the number in the $\operatorname{SNB} \operatorname{SWS} 1(q, p, n)$ switching fabric as $\frac{q^{2}-1}{q-1} \leq m_{\max } \leq \frac{n q}{2 q-1}$.

\subsection{RPNB SWS1 $(q, p, n)$ Switching Fabrics with $2 \leq m_{\max } \leq n$}

Given an $\operatorname{SWS1}(q, p, n)$ switching fabric with $p \geq q^{2}$ for $m$-slot connections, $1 \leq m \leq m_{\max }$ and $2 \leq m_{\max } \leq n$, from Property 1 , we can see that only under the condition that connections depart will a blocking state arise. Recall that time is less critical for connection rearrangement during departures than during arrivals. Thus, for RPNB, rather than rearranging existing connections once a blocking state has occurred, rearrangement is done immediately after a departure. In other words, while a connection, say $(i, j, m)$, departs, Routing Algorithm 2 (for Rearrangements) is applied immediately. From Property 1 and Theorem 2, Theorem 3, given below, can be derived immediately.

Theorem 3. An SWS1 $(q, p, n)$ switching fabric for $m$-slot connections with $1 \leq m \leq m_{\max }$ and $2 \leq m_{\max } \leq n$ is $R P N B$ if $p \geq q^{2}$.

Proof. The theorem holds because of Property 1 and Theorem 2, while existing connections, that is, $\left(i, j^{\prime}, m\right)$ and $\left(i^{\prime}, j, m\right)$ for $1 \leq i^{\prime}, j^{\prime} \leq q$, are rearranged by Routing Algorithm 2 (for Rearrangements) after connection $(i, j, m)$ departs.

Similar to Theorem 2, from Theorem 3, we can see that the proposed RPNB SWS1 $(q, p, n)$ switching fabric with $2 \leq m_{\max } \leq n$ requires fewer middle switches than the SNB SWS1 $(q, p, n)$ switching fabric given in [7] as $\frac{q^{2}-1}{2(q-1)} \leq m_{\max } \leq \frac{n q}{2 q-1}$ (Equation (1)). Thus, the upper bound of the RPNB SWS1 $(q, p, n)$ switching fabric for $m$-slot connections, $1 \leq m \leq m_{\max }$ and $2 \leq m_{\max } \leq n$, reduces by around half the number of middle switches relative to the number in the $\operatorname{SNB} \operatorname{SWS}(q, p, n)$ switching fabric as $\frac{q^{2}-1}{q-1} \leq m_{\max } \leq \frac{n q}{2 q-1}$. It is worth noting that even though the $\operatorname{RPNB}$ and $\operatorname{RNB} \operatorname{SWS} 1(q, p, n)$ switching fabrics have the same results, the former does connection rearrangements during departures, which requires less critical time than during arrivals.

\section{Conclusions}

Elastic optical networks flexibly allocate bandwidth to a connection to improve utilization efficiency. The paper considers an S-W-S switching fabric, represented as SWS1 $(q, p, n)$, for elastic optical networks. RNB and RPNB SWS1 $(q, p, n)$ switching fabrics are proposed in this paper. The number of middle switches is calculated and evaluated. Note that for an $\operatorname{SWS} 1(q, p, n)$ switching fabric with given parameters $q$ and $n$, reducing the value of $p$, namely, the number of middle switches, leads to lowering the hardware cost, area requirements, and power consumption. The proposed RNB and RPNB $\operatorname{SWS1}(q, p, n)$ switching fabrics for $m$-slot connections where $1 \leq m \leq m_{\max }$ with either $m_{\max }=1$ or $\frac{q^{2}-1}{q-1} \leq m_{\max } \leq \frac{n q}{2 q-1}$ consume around half the number of middle switches compared to the SNB $\operatorname{SWS1}(q, p, n)$ switching fabric given in [7]. Different from the SNB SWS1 $(q, p, n)$ switching fabric, connection rearrangements are required for both RNB and $\operatorname{RPNB} \operatorname{SWS1}(q, p, n)$ switching fabrics. Though the RPNB SWS1 $(q, p, n)$ switching fabric has the same result in terms of $p$ as the RNB SWS1 $(q, p, n)$ switching fabric, the former does connection rearrangements during departures, which requires less critical time than during arrivals. It is worth noting that some rearrangement methods adopted for the RNB and RPNB SWS1 $(q, p, n)$ switching fabrics could cause connection delays, and they may need some kind of setup network that would consume more power. All of these factors could affect the performance and throughput of the proposed switching fabrics. These factors, as well 
as further improvement of the upper bounds for RNB and RPNB SWS1 $(q, p, n)$ switching fabrics, will be investigated in the future.

Funding: This work was supported by the Ministry of Science and Technology, Taiwan, under Contract MOST 108-2221-E-024-002-MY2.

Conflicts of Interest: The authors declare no conflict of interest.

\section{References}

1. Jinno, M.; Takara, H.; Kozicki, B.; Tsukishima, Y.; Sone, Y.; Matsuoka, S. Spectrum-efficient and scalable elastic optical path network: Architecture, benefits, and enabling technologies. IEEE Commun. Mag. 2009, 47, 66-73. [CrossRef]

2. Gerstel, O.; Jinno, M.; Lord, A.; Yoo, S.J.B. Elastic optical networking: A new dawn for the optical layer? IEEE Commun. Mag. 2012, 50, S12-S20. [CrossRef]

3. Tomkos, I.; Azodolmolky, S.; Sole-Pareta, J.; Careglio, D.; Palkopoulou, E. A tutorial on the flexible optical networking paradigm: State of the art, trends, and research challenges. Proc. IEEE 2014, 102, 1317-1337. [CrossRef]

4. Spectral Grids for WDM Applications: DWDM Frequency Grid. In ITU-T Standard G.694.1; International Telecommunications Union (CCIR) and (CCITT): Geneva, Switzerland, 2012.

5. Zhang, P.; Li, J.; Guo, B.; He, Y.; Chen, Z.; Wu, H. Comparison of node architectures for elastic optical networks with waveband conversion. China Commun. 2013, 10, 77-87.

6. Chen, Y.; Li, J.; Zhu, P.; Niu, L.; Xu, Y.; Xie, X.; He, Y.; Chen, Z. Demonstration of Petabit scalable optical switching with subband-accessibility for elastic optical networks. In Proceedings of the 2014 OptoElectronics and Communication Conference and Australian Conference on Optical Fibre Technology C, Melbourne, Vic, Australia, 6-10 July 2014.

7. Danilewicz, G.; Kabaciński, W.; Rajewski, R. Strict-sense nonblocking space-wavelength-space switching fabrics for elastic optical network nodes. J. Opt. Commun. Netw. 2016, 8, 745-756. [CrossRef]

8. Kabaciński, W.; Michalski, M.; Rajewski, R. The strict-sense nonblocking W-S-W node architectures for elastic optical switch. J. Lightwave Technol. 2016, 34, 3155-3162. [CrossRef]

9. Kabaciński, W.; Michalski, M.; Abdulsahib, M. The strict-sense nonblocking elastic optical switch. In Proceedings of the 2015 IEEE 16th International Conference on High Performance Switching and Routing (HPSR), Budapest, Hungary, 1-4 July 2015.

10. Kabaci'nski, W.; Michalski, M.; Abdulsahib, M. Wide-sense nonblocking elastic optical switch. Opt. Switch. Netw. 2016, 25, 71-79. [CrossRef]

11. Kabaciński, W.; Rajewski, R.; AI-Tameemi, A. Simultaneous connections routing in W-S-W elastic optical switches with limited number of connection rates. In Proceedings of the 2017 International Conference on Optical Network Design and Modeling (ONDM), Budapest, Hungary, 15-18 May 2017.

12. Lin, B.-C. Rearrangeable W-S-W elastic optical networks generated by graph approaches. J. Opt. Commun. Netw. 2018, 10, 675-685. [CrossRef]

13. Yoo, S.J.B. Integrated photonic-electronic technologies for next generation data centers and the future internet. In Proceedings of the 2012 International Conference on Photonics in Switching (PS), Ajaccio, France, 11-14 September 2012.

14. Clos, C. A study of non-blocking switching networks. Bell Syst. Tech. J. 1953, 32, 406-424. [CrossRef]

15. Hwang, F. The Mathematical Theory of Nonblocking Switching Networks; World Scientific: Singapore, 2004.

16. Jajszczyk, A.; Jekel, G. A new concept-repackable networks. IEEE Trans. Commun. 1993, 41, $1232-1237$. [CrossRef]

17. Jajszczyk, A. Nonblocking, repackable, and rearrangeable Clos networks: Fifty years of the theory evolution. IEEE Commun. Mag. 2003, 41, 28-33. [CrossRef]

18. Chakrabarty, A.; Collier, M. Efficient Implementation of Symmetric Multistage Repackable Networks. In Proceedings of the 2010 International Conference on Computational Intelligence and Communication Networks, Bhopal, India, 26-28 November 2010. 
19. Lin, B.-C.; Lea, C.-T. Repackable multi- $\log _{2} N$ networks. Theor. Comput. Sci. 2016, 609, 22-31. [CrossRef]

20. West, D.B. Introduction to Graph Theory; Pearson: New York City, NY, USA, 2001.

(C) 2020 by the author. Licensee MDPI, Basel, Switzerland. This article is an open access article distributed under the terms and conditions of the Creative Commons Attribution (CC BY) license (http://creativecommons.org/licenses/by/4.0/). 